\title{
Nanotechnology applied to Reneweable Energy
}

\section{Nanotechnology applied to Reneweable Energy}

\author{
Juan Alberto Argüello García-Pertusa*, Mohammed Alanbari, ${ }^{* *}$,isbel Cerpa ${ }^{* *}$ \\ *UNIVERSIDAD EUROPEA DE MADRID. Departamento Óptica Universidad Europea - Escuela Politécnica C/ Tajo s/n. \\ 28670 Villaviciosa de Odón. Madrid,- España. Tfno. (+34) 91- 2115658 - www.uem.es. juanalberto.arguello@uem.es \\ ** UNIVERSIDAD EUROPEA DE MADRID, Departamento INGENIERÍA INDUSTRIAL Universidad \\ Europea - Escuela Politécnica C/ Tajo s/n. 28670 Villaviciosa de Odón. Madrid,- España.Tfno. (+34) 91- 211 \\ 5658 - www.uem.es.
}

Abstract- Lately, an important application for Nanotechnology is renewable energy. Scientists and engineers have discovered that by using this technology efficiency, cost, size, and weight are improved directly, and the environment and green energy are improved indirectly.

Benefits such as these make the investment of capital in the research and development of nanotechnology a top priority element, such as in the strategic European plan.

An important subfield of nanotechnology related to energy is nanofabrication. Nanofabrication is the process of designing and creating devices on the nanoscale. Creating devices smaller than 100 nanometers opens many doors for the development of new ways to capture, store, and transfer energy. The inherent level of control that nanofabrication could give scientists and engineers would be critical in solving many of the problems the world is facing today with the current generation of energy technologies. Another current problem is the cost of nanofabrication.

Keywords- nanotechnology; renewable energy; photovoltaic; wind power; thermal solar

\section{NANOTECHNOLOGY RENEWEABLE ENERGY}

AND

Nanotechnology operates at such a fundamental level that there is very little of a technological nature that it will not impact. Thus its effects on energy generation, transmission, storage and consumption are numerous and diverse. Some will be incremental and some quite possibly revolutionary.

Nanotechnology helps increase the efficiency of existing forms of energy while opening up completely new ways of exploiting renewable energies. Given its role as a key and cross-sectional technology, nanotechnology has the potential to achieve decisive technological breakthroughs in the energy sector, thus making important contributions to sustainable energy supplies.
The relevant innovations apply across the entire value-added chain. Innovative methods will boost efficiency in all subareas: These include tapping energy sources such as crude oil, natural gas and coal as well as renewable energy sources such as geothermal heat, the sun, wind, water, the tides and biomass. There are also solutions for generating (energy transformation), transmitting and storing electricity all the way to electricity consumption.

The number of patents issued for renewable-energy technologies has risen sharply over the last decade, according to new research from MIT and the Santa Fe Institute (SFI). The study shows that investments in research and development, as well as in the growth of markets for these products, have helped to spur this dramatic growth in innovation. [1]

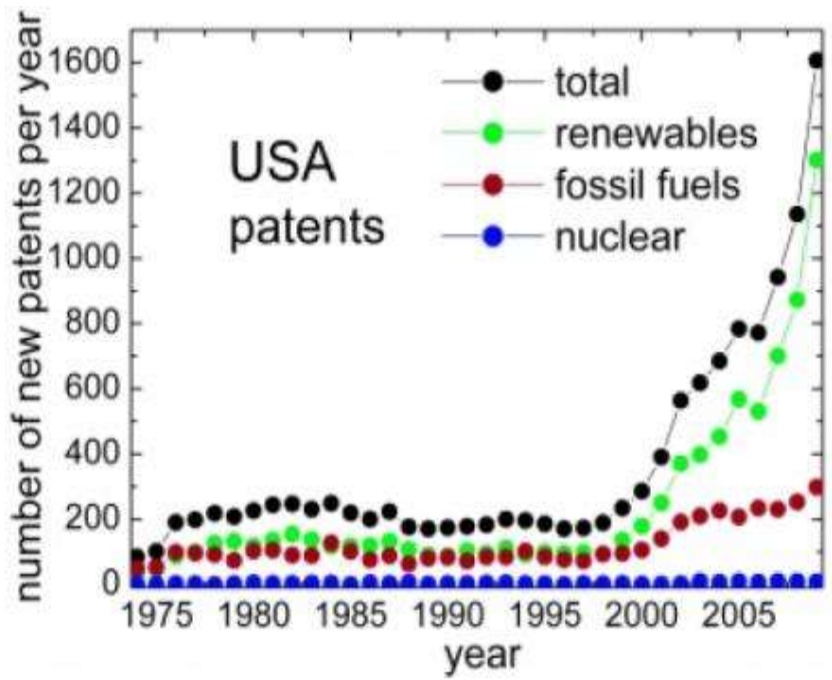

Figure 1. Study found a dramatic increase in the overall number of energy-related patents issued in the U.S., with increase in renewable energy patents far outpacing those in other energy sectors. A similar trend was seen globally.

The inventory is an essential resource for consumers, citizens, policymakers, and others who are interested in 
learning about how nanotechnology is entering the marketplace. It is meant to be international and expanding. As of March 10, 2011, the nanotechnology consumer products inventory contained 1317 products or product lines.

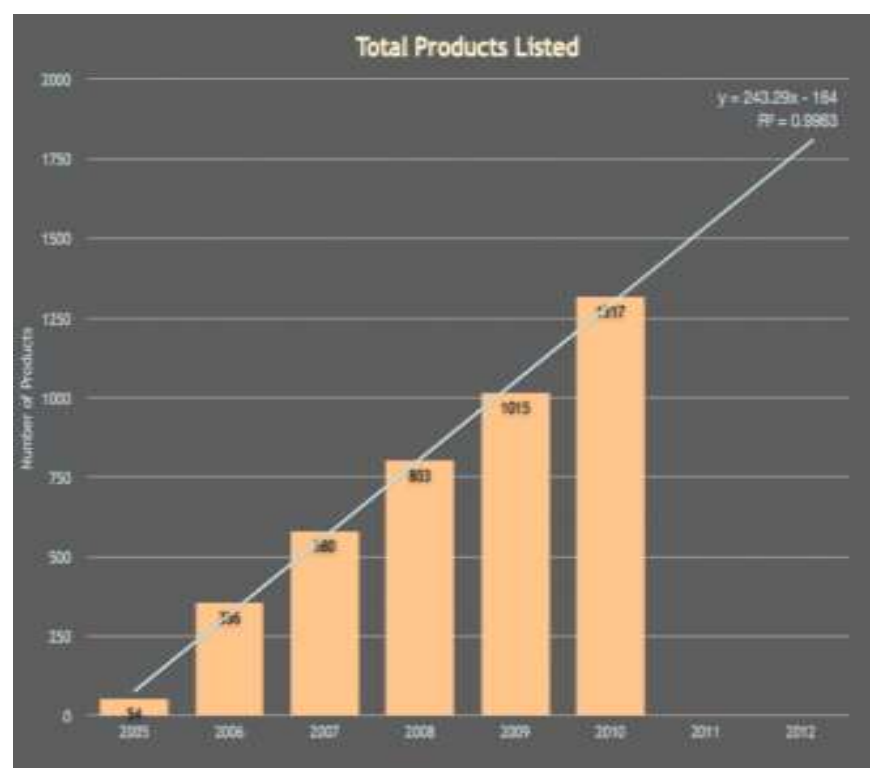

Figure 2. Number of total products listed, by date of inventory update, with regression analysis. [2]

\section{PHotovoltaic}

The fundamental objectives of nanotechnology applied to solar cells are:

- Increase the light absorption rate.

- Increase the efficiency of the photovoltaic effect.

- Decrease the cost of manufacturing.

- Make PV cells adaptable to different scales and localities.

Nanotechnology is already used to provide improved performance coatings for photovoltaic (PV) and solar thermal panels. Hydrophobic and self-cleaning properties combine to create more efficient solar panels, especially during inclement weather. PV covered with nanotechnology coatings are said to stay cleaner for longer to ensure maximum energy efficiency is maintained.

Using nanoparticles in the manufacture of solar cells has the following benefits:

- Reduced manufacturing costs as a result of using a low temperature process similar to printing instead of the high temperature vacuum deposition process typically used to produce conventional cells made with crystalline semiconductor material.
- Reduced installation costs achieved by producing flexible rolls instead of rigid crystalline panels. Cells made from semiconductor thin films will also have this characteristic.

- Currently available nanotechnology solar cells are not as efficient as traditional ones, however their lower cost offsets this. In the long term, nanotechnology versions should both be lower cost and, using quantum dots, should be able to reach higher efficiency levels than conventional ones.

\section{Increasing the efficiency of energy production}

Nanotechnology could potentially have a great impact on clean energy production. Research is underway to use nanomaterials for purposes including more efficient solar cells, practical fuel cells, and environmentally friendly batteries. The most advanced nanotechnology projects related to energy are: storage, conversion, manufacturing improvements by reducing materials and process rates, energy saving (by better thermal insulation for example), and enhanced renewable energy sources.

Today's best solar cells have layers of several different semiconductors stacked together to absorb light at different energies but they still only manage to use 40 percent of the Sun's energy. Commercially available solar cells have much lower efficiencies (15-20\%). Nanotechnology could help increase the efficiency of light conversion by using nanostructures.

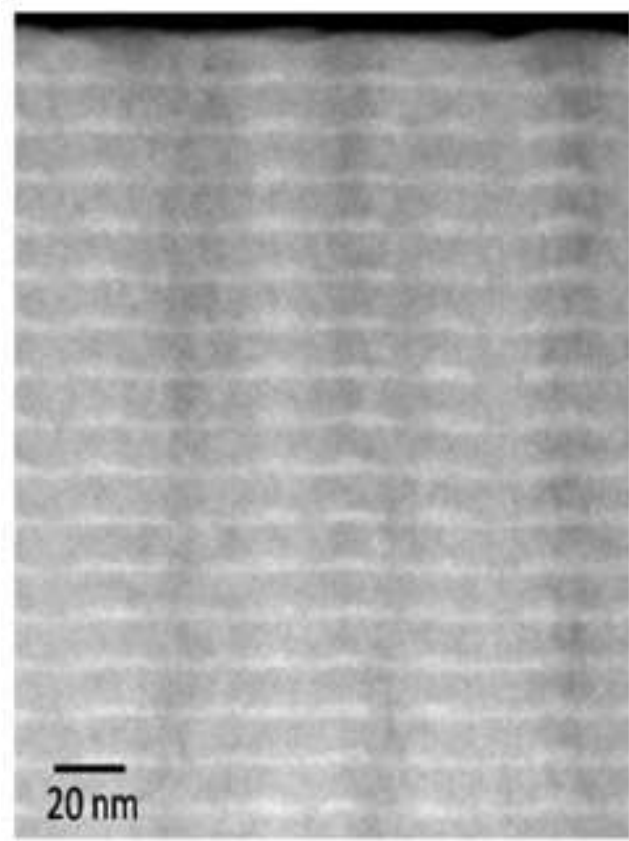

TEM image of self-organized quantum dot superlattice 


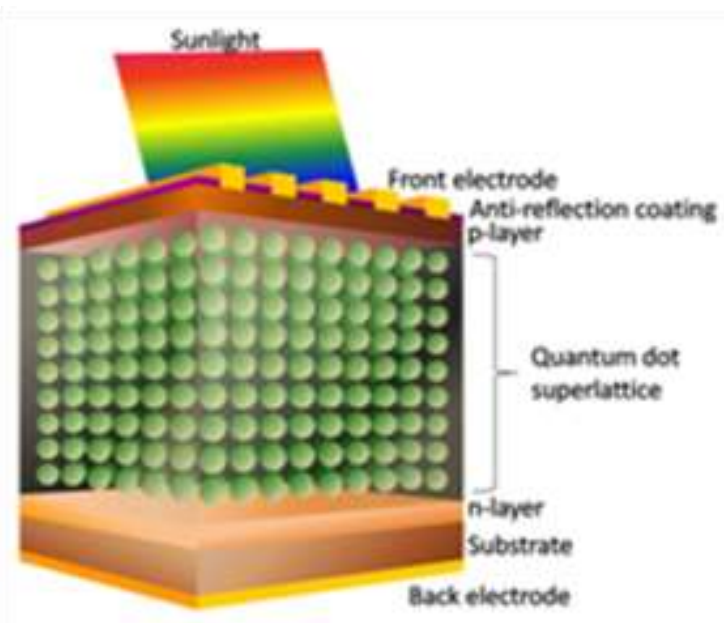

\section{Schematic structure of quantum dot intermediate band solar cell}

New semiconductor materials and new quantum nanostructures are produced by crystal growth using molecular beam epitaxy to fabricate high-efficiency nextgeneration solar cells, such as multi-junction tandem solar cells and intermediate band solar cells.

A 3-dimensional quantum dot superlattice which consists of multi-stacked layers of self-organaized InAs quantum dots is a promising absorber for intermediate band solar cells.

This increases the efficiency to $35 \%$ compared to $16 \%$ of silicium.

\section{Advances in Solar Panel Nanotechnology Self-Cleaning Solar Panels}

In July 2012, ecoSolargy launched a range of solar panels which use self-cleaning glass - this keeps the panels free of dirt and debris, to ensure that the PV cells receive as much of the incident solar energy as possible. This technique is very effective in helping the conventional cells to perform at peak efficiency.

We will probably see much more of this sort of indirect application of nanotechnology to solar panels in the coming years, before nanotechnology is able to gain a significant market share in the actual PV materials used.

\section{Dye-Enhanced Solar Cells - Mimicking Photosynthesis}

In May 2012, Northwestern University researchers developed a new sort of "dye-sensitized" solar cell (part of the second generation of photovoltaic technologies), which uses an organic dye monolayer to help absorb sunlight, much as plants do for photosynthesis.
Dye-sensitized PV cells have been explored before however, the organic dye used is usually a liquid, which can leak out, drastically shortening the lifetime of the cell. The advance made by the team at Northeastern University is to use a dye which is just as effective at capturing solar energy, but solidifies, preventing it from leaking and giving the cell a viable lifetime.

Their novel solar cell also uses a number of other nanomaterials, like titanium dioxide nanoparticles and caesium tin iodide thin films, as high-performance p-type and n-type semiconductors.

\section{Connection Between Stacked Solar Cells [3]}

North Carolina State University researchers have come up with a new technique for improving the connections between stacked solar cells, which should improve the overall efficiency of solar energy devices and reduce the cost of solar energy production.

Stacked solar cells consist of several solar cells that are stacked on top of one another. Stacked cells are currently the most efficient cells on the market, converting up to 45 percent of the solar energy they absorb into electricity.

But to be effective, solar cell designers need to ensure the connecting junctions between these stacked cells do not absorb any of the solar energy and do not siphon off the voltage the cells produce -- effectively wasting that energy as heat.

\section{Nanowire Solar Cells Raise Efficiency Limit [4]}

Scientists from the Nano-Science Center at the Niels Bohr Institut, Denmark and the Ecole Polytechnique Fédérale de Lausanne, Switzerland, have shown that a single nanowire can concentrate the sunlight up to 15 times of the normal sun light intensity. The results are surprising and the potential for developing a new type of highly efficient solar cells is great.

Due to some unique physical light absorption properties of nanowires, the limit of how much energy we can utilize from the sun's rays is higher than previous believed. These results demonstrate the great potential of development of nanowire-based solar cells.

During recent years research groups have studied how to develop and improve the quality of nanowire crystals, which are a cylindrical structure 10,000 times smaller than a human hair. The nanowires are predicted to have great potential in the development not only of solar cells, but also of future quantum computers and other electronic products. 


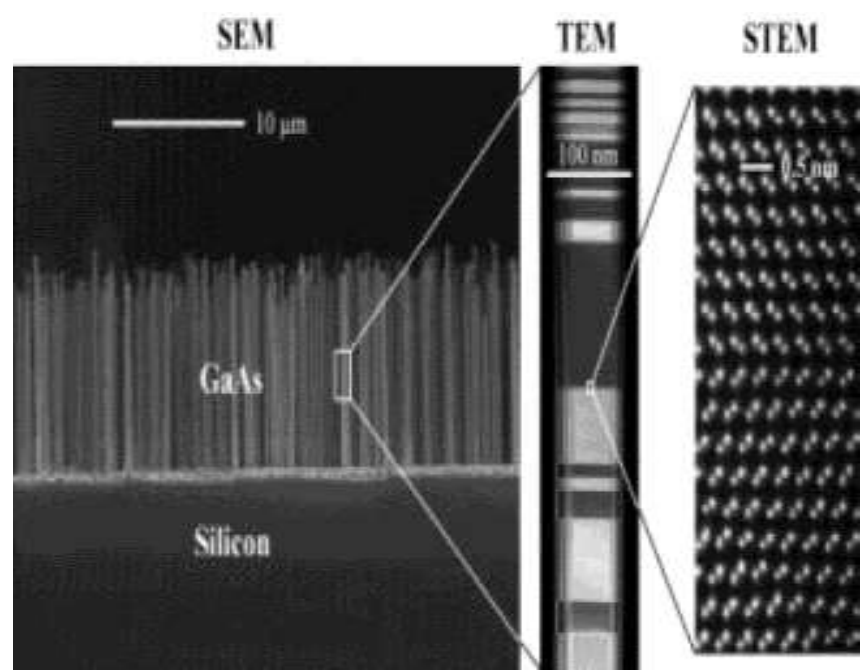

It turns out that the nanowires naturally concentrate the sun's rays into a very small area. Because the diameter of a nanowire crystal is smaller than the wavelength of the light coming from the sun it can cause resonances in the intensity of light in and around nanowires. Thus, the resonances can create concentrated sunlight, where the energy is converted, which can be used to give a higher conversion efficiency of the sun's energy.

\section{Quantum dots or nanowires [5]}

Putting the amorphous quantum dots in an array or merging them into a nanowire are the best assemblies for maximizing the efficiency of silicon nanomaterials to absorb light and transport charge throughout a photovoltaic system according to a study from researchers at North Dakota State University and the University of South Dakota.

Amorphous Silicon nanowire facilitates harvesting of solar energy in the form of a photon. In the process of light absorption a pair of mobile charge carriers is created. The energy of their directed motion is then transformed into electricity. Electron and hole charge distributions are often located in different regions of space due to multiple structural defects in amorphous silicon nanowires.

\section{The crown jewel of nanotechnology [6]}

Graphene has extreme conductivity and is completely transparent while being inexpensive and nontoxic. This makes it a perfect candidate material for transparent contact layers for use in solar cells to conduct electricity without reducing the amount of incoming light -- at least in theory. Whether or not this holds true in a real world setting is questionable as there is no such thing as "ideal" graphene -a free floating, flat honeycomb structure consisting of a single layer of carbon atoms: interactions with adjacent layers can change graphene's properties dramatically.

Now the HZB Institute for Silicon Photovoltaics has shown that graphene retains its impressive set of properties when it is coated with a thin silicon film. These findings have paved the way for entirely new possibilities to use in thin-film photovoltaics.

Graphene was deposited onto a glass substrate. The ultrathin layer is but one atomic layer thick (0.3 Angström, or 0.03 nanometers), although charge carriers are able to move about freely within this layer. This property is retained even if the graphene layer is covered with amorphous or polycrystalline silicon.

Their measurements of carrier mobility using the Hall-effect showed that the mobility of charge carriers within the embedded graphene layer is roughly 30 times greater than that of conventional zinc oxide based contact layers.

[7] Also graphene can be applied as a substitute for platinum when Dye-sensitized solar cells are thin, flexible, easy to make and very good at turning sunshine into electricity. However, a key ingredient is one of the most expensive metals on the planet: platinum. While only small amounts are needed, at $\$ 1,500$ an ounce, the cost of the silvery metal is still significant.

\section{All-Carbon Solar Cell [8]}

The solar cell consists of a photoactive layer, which absorbs sunlight, sandwiched between two electrodes. In a typical thin film solar cell, the electrodes are made of conductive metals and indium tin oxide (ITO). And this is replaced with conventional electrodes with graphene -- sheets of carbon that are one atom thick -and single-walled carbon nanotubes that are 10,000 times narrower than a human hair.

The active layer made of carbon nanotubes and "buckyballs" -- soccer ball-shaped carbon molecules just one nanometer in diameter. Currently the efficiency is less than $1 \%$.

\section{III.SOLAR THERMAL}

We can apply almost the same nanotechnology from the second chapter to this kind of generation energy.

As is well known, for example one of the most important problems is that this type of energy is the heliostats need to clean almost every day because they get dirty. Therefore, it's important to have a self-cleaning system. This isn't an easy thing to do, because you need specific machines, water and other chemical products.

To resolve this problem we can use the lotus-leaf effect using nanotechnology to reproduce what nature does.

Self-cleaning surfaces are based on the superhydrophobic effect, which causes water droplets to roll off with ease, carrying away dirt and debris.

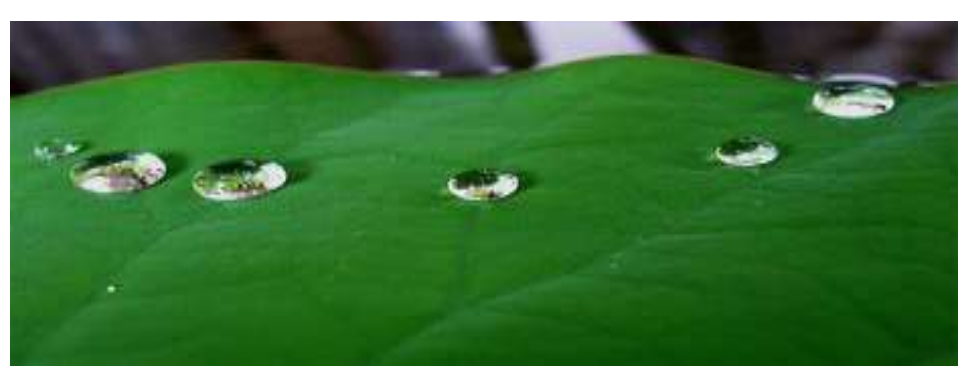


Every material has an energy associated with its surface, and when a fluid droplet is in contact with this surface, the energies of the three-phase contact line balance to a minimum, forming a distinct angle of contact with the other surface.

A specific research project for this type of energy is the Thermalcond project, which is a European collaborative project aimed at developing a novel collector containing plastic components with high efficiency, low cost and less weight structures compared to metallic collectors.

These collectors will have an improved design due to the use of plastic materials, high thermal conductive nanoparticles modified with SAM technology, and a flexible absorber coating based on nanometallic oxides.

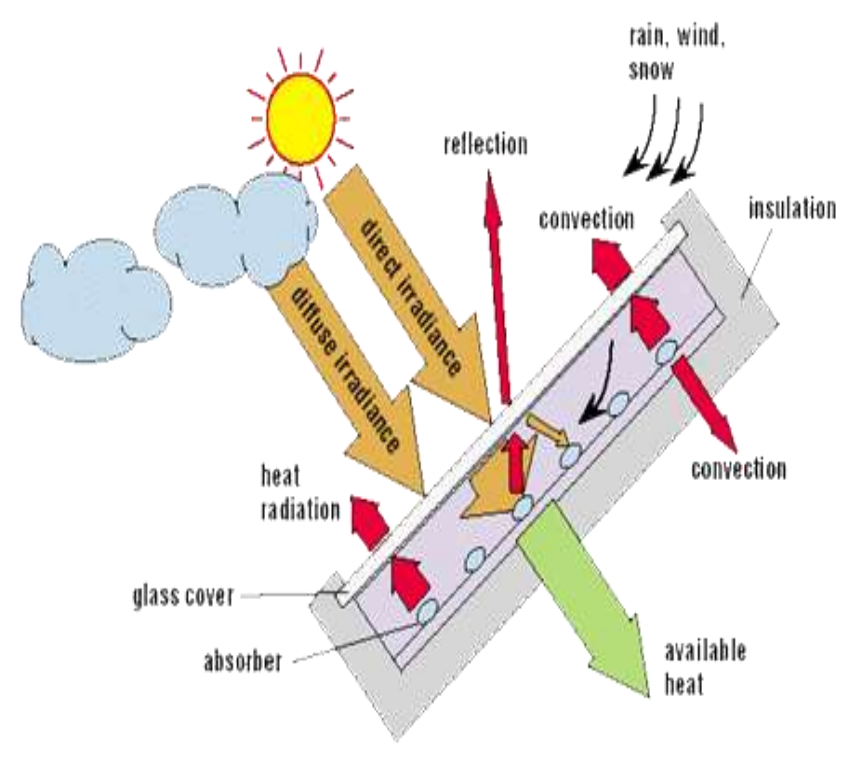

IV.WIND POWER

Various nanotechnologies are utilized to improve the efficiency of power-generating wind turbines, including nanoparticle-containing lubricants that reduce the friction generated from the rotation of the turbines, nanocoatings for de-icing and self-cleaning technologies, and advanced nanocomposites that provide lighter and stronger wind blades. For example the following:

-De-icing coatings

- Surface treatment to repel water could prevent ice forming on the blades.

- Lotus Plant non-wettable self-cleaning leaves due to nanostuctured rough surface with wax crystals.

- Reduced porosity and moisture absorbance

- Self-cleaning coatings like Pilkington's selfcleaning glass

-Weight saving
- Strength/ weight improvements

- Tensile strength up $40 \%$

- Tensile modulus (elasticity) up $68 \%$

- Flexural strength up $60 \%$

- Flexural modulus (bending) up 126\%

- Distortion temperature up from $65 \%$ to $152 \%$

- Improved flame retardant properties

- $20 \%$ weight saving over conventional materials -Lubricants

- Nanolubricant for improved wear resistance at all temperatures and pressures

-New Sealants

- Novel sealants based on nanocomposite elastomers

-Hydraulic systems

- Brake systems, pitch \& yaw controls

- On-board cranes, locking systems.

- Pumps, drives, oil tanks, filters, pressure valves and control systems

-Power pack improvements

- Carbon nanotubes as fuel storage

- Control systems are increasingly important

- SCADA for systems required by grid operators -Condition monitoring

- Remote control and full monitoring

- Mini generators and energy storage for start-up and nil wind.

\section{CONCLUSIONS}

Clean power generation from renewable energy is seen as the solution to the climate impacts of the energy sector. However, there are barriers yet to be overcome to scale up energy production through renewables.

In the United States, only 9 percent of total energy consumed came from renewable sources in 2011 according to the Institute for Energy Research. In Germany, renewables only account for 12 percent.

The main challenges now for the application of nanomaterials in the energy sector are the improvement of efficiency, reliability, safety and lifetime, as well as the reduction of costs.

To date, universities, research institutes and even governments are paying attention to the synergy that could be established between nanotechnology and renewable energy.

Thanks to better nanomaterials, PV solar cells are increasing their efficiency while reducing their manufacturing and electricity production costs at an unprecedented rate. Hydrogen production, storage and transformation into electricity in fuel cells are being benefited from more efficient catalysts for water splitting, better nanostructured materials for higher hydrogen adsorption capacity and cheaper, simpler fuel cells. 


\section{REFERENCES}

[1] Béla Nagy, J. Doyne Farmer, Quan M. Bui, Jessika E. Trancik. Statistical Basis for Predicting Technological $\begin{array}{lllll}\text { Progress. PLoS } & \text { ONE, } & \text { 2013; } & 8 & \text { (2): }\end{array}$ DOI:10.1371/journal.pone.0052669

[2] http://www.nanotechproject.org/inventories/consumer/

[3] Joshua P. Samberg, C. Zachary Carlin, Geoff K. Bradshaw, Peter C. Colter, Jeffrey L. Harmon, J. B. Allen, John R. Hauser, S. M. Bedair. Effect of GaAs interfacial layer on the performance of high bandgap tunnel junctions for multijunction solar cells. Applied Physics Letters, 2013; 103 (10): 103503 DOI: 10.1063/1.4819917

[4] Peter Krogstrup, Henrik Ingerslev Jørgensen, Martin Heiss, Olivier Demichel, Jeppe V. Holm, Martin Aagesen, Jesper Nygard, Anna Fontcuberta i Morral. Singlenanowire solar cells beyond the Shockley-Queisser limit. Nature Photonics, 2013; DOI: $10.1038 /$ nphoton.2013.32

[5] Andrei Kryjevski, Dmitri Kilin, Svetlana Kilina. Amorphous silicon nanomaterials: Quantum dots versus nanowires.Journal of Renewable and Sustainable Energy, 2013; 5 (4): 043120 DOI: 10.1063/1.4817728

[6] M. A. Gluba, D. Amkreutz, G. V. Troppenz, J. Rappich, N. H. Nickel. Embedded graphene for large-area siliconbased devices. Applied Physics Letters, 2013; 103 (7): 073102 DOI: $10.1063 / 1.4818461$

[7] Hui Wang, Kai Sun, Franklin Tao, Dario J. Stacchiola, Yun Hang Hu. 3D Honeycomb-Like Structured Graphene and Its High Efficiency as a CounterElectrode Catalyst for Dye-Sensitized Solar Cells. Angewandte Chemie, 2013; DOI: $10.1002 /$ ange. 201303497

[8] Marc P. Ramuz, Michael Vosgueritchian, Peng Wei, Chenggong Wang, Yongli Gao, Yingpeng Wu, Yongsheng Chen, Zhenan Bao. Evaluation of Solution-Processable Carbon-Based Electrodes for All-Carbon Solar Cells.ACS Nano, 2012; 121031083325001 DOI: $10.1021 / \mathrm{nn} 304410 \mathrm{w}$ 